\title{
Über eine Klasse von kubischen diophantischen Gleichungen mit drei Unbekannten
}

\author{
Von Stig Christofferson
}

\section{§ 1. Einleitung}

Wir betrachten die diophantische Gleichung

$$
u^{3}+a b^{2} v^{3}+a^{2} b w^{3}-3 a b u v w=C,
$$

wo $a$ und $b$ natürliche Zahlen sind, deren Produkt $a b>1$ durch kein Primzahlquadrat teilbar ist, und $C$ eine natürliche Zahl ist.

Wenn die ganzen rationalen Zahlen $u, v, w$ die Gleichung (1) befriedigen, so wird die Zahl

mit

$$
\begin{gathered}
\omega=u+v \alpha+w \beta, \\
\alpha=\sqrt[3]{a b^{2}} \text { und } \beta=\sqrt[3]{a^{2} b},
\end{gathered}
$$

eine Lösung von (1) genannt. $\alpha$ und $\beta$ sollen die reellen dritten Wurzeln sein, und sie sind also nach der Voraussetzung über $a$ und $b$ positive irrationale Zahlen. Es ist keine Einschränkung, wenn wir im folgenden $a>b$ voraussetzen, dann wird $\alpha<\beta$. Eine Lösung $\omega$ heisst grösser als eine andere Lösung $\omega_{1}$, wenn von der Zahlen $\omega$ und $\omega_{1}$ die erstere die grössere ist, und umgekehrt nennt man dann $\omega_{1}$ kleiner als $\omega$. Zwei Lösungen $\omega$ und $\omega_{1}$ heissen gleich, wenn $\omega=\omega_{1}$, was nur für $u=u_{1}, v=v_{1}, w=w_{1}$ vorkommt.

In dem reinen kubischen Zahlkörper $\boldsymbol{K}(\alpha)$ ist die Form

$$
F(u, v, w)=u^{3}+a b^{2} v^{3}+a^{2} b w^{3}-3 a b u v w
$$

einfach die Norm der Zahl $\omega=u+v \alpha+w \beta$, und wenn $\varrho$ und $\varrho^{2}$ die primitiven kubischen Einheitswurzeln sind, $\varrho^{2}+\varrho+1=0$, wird, mit

$$
\begin{gathered}
\omega^{\prime}=u+v \alpha \varrho+w \beta \varrho^{2} \text { and } \omega^{\prime \prime}=u+v \alpha \varrho^{2}+w \beta \varrho, \\
F(u, v, w)=\omega \omega^{\prime} \omega^{\prime \prime}
\end{gathered}
$$

Wir setzen

$$
\begin{gathered}
\omega^{\prime} \omega^{\prime \prime}=\bar{\omega}=\bar{u}+\bar{v} \alpha+\bar{w} \beta, \\
\bar{u}=u^{2}-a b v w, \quad \bar{v}=a w^{2}-u v, \quad \bar{w}=b v^{2}-u w .
\end{gathered}
$$


S. CHRISTOFFERSON, Kubische diophantische Gleichungen mit drei Unbekannten

Die Form $F(u, v, w)$ kann damit als Produkt von zwei Faktoren geschrieben werden :

$$
F(u, v, w)=(u+v \alpha+w \beta)(\bar{u}+\bar{v} \alpha+\bar{w} \beta) .
$$

Mit $u, v, w$ sind auch $\bar{u}, \bar{v}, \bar{w}$ ganz rational. $F(u, v, w)$ kann gleich 0 sein, nur wenn $u=v=w=0$, denn $u+v \alpha+w \beta=u+v \alpha+w b^{-1} \alpha^{2}$, und das Polynom $x^{3}-a b^{2}$ ist im rationalen Zahlkörper irreduzibel. $\bar{\omega}=0$ hat auch $u=v=w=0$ zur Folge. Uns interessiert also nur der Fall $C \neq 0$, und wegen $F(u, v, w)=-F(-u,-v,-w)$ genügt es, positive $C$ zu betrachten.

$\omega^{\prime}$ und $\omega^{\prime \prime}$ sind nach (3) imaginärkonjugiert, d.h. $\bar{\omega}=\omega^{\prime} \omega^{\prime \prime}$ ist stets nichtnegativ, und für positives $C$ muss also eine Lösung $\omega$ von (1) positiv sein.

Wir bemerken auch, dass

mit

$$
(x+y \alpha+z \beta)(u+v \alpha+w \beta)=u_{1}+v_{1} \alpha+w_{1} \beta
$$

$$
\left\{\begin{array}{l}
u_{1}=x u+a b y w+a b z v \\
v_{1}=x v+y u+a z w \\
w_{1}=x w+b y v+z u
\end{array}\right.
$$

Daraus folgt, dass ein Produkt von einer endlichen Anzahl von Zahlen des Typus (2) eine Zahl desselben Typus ist, und wenn wir der Kürze halber

setzen, so folgt

$$
F(\omega)=F(u, v, w)
$$

$$
F\left(\omega \omega_{1}\right)=F(\omega) F\left(\omega_{1}\right)
$$

weil $\omega \omega_{1}\left(\omega \omega_{1}\right)^{\prime}\left(\omega \omega_{1}\right)^{\prime \prime}=\omega \omega^{\prime} \omega^{\prime \prime} \omega_{1} \omega_{1}^{\prime} \omega_{1}^{\prime \prime}$.

Aus (5) folgt auch, wenn man $x+y \alpha+z \beta=\bar{u}+\bar{v} \alpha+\bar{w} \beta$ setzt,

$$
\left\{\begin{aligned}
u \bar{u}+a b v \bar{w}+a b w \bar{v} & =F(u, v, w), \\
u \bar{v}+v \bar{u}+a w \bar{w} & =0 \\
u \bar{w}+b v \bar{v}+w \bar{u} & =0
\end{aligned}\right.
$$

Die Existenz von unendlich vielen Lösungen der Gleichung

$$
u^{3}+D v^{3}+D^{2} w^{3}-3 D u v w=1
$$

wenn $D$ kein Kubus einer ganzen Zahl ist, folgt natürlich aus der allgemeinen Theorie der Einheiten in algebraischen Zahlkörpern. Mehrere Verfasser haben sich mit der numerischen Berechnung von Lösungen der Gleichung (8) beschäftigt. In Arbeiten von Markoff [1] ${ }^{1}$, Merssel [2], Dedekind [3], Nagell [4], Wolfe [5], Cassels [6], und Selmer [7] findet man Tafeln von Lösungen für kleine Werte von $D$. Bei Nagell, Cassels und Selmer geben die Lösungen im allgemeinen die Fundamentaleinheiten in entsprechenden Körpern. SELMER hat ausserdem

1 Die Zahlen in eckigen Klammern verweisen auf das Literaturverzeichnis am Ende der Arbeit. 
Tafeln für $D \leq 250$ und für $D$ gleich ungeraden Vielfachen von 4 bis nach $D \leq 400$ in Aussicht gestellt.

Der Zweck dieser Arbeit ist, einige elementare Abschätzungen der Lösungen von (1) herzuleiten, und zunächst wird eine von DIRICHLET [8] angegebene Beweismethode so ausgenutzt, dass sie eine Abschätzung einer Lösung für $C=1$ ermöglicht.

\section{§ 2. Hilfssätze}

Hilfssatz 1. Die Ungleichung

$$
0<F(u, v, w)<3 \alpha^{2}+5 \alpha \beta+3 \beta^{2}
$$

hat unendlich viele Lösungen in ganzen Zahlen $u, v, w$.

Beweis. Wenn $t$ eine natürliche Zahl ist, und $v$ und $w$ die Zahlen $0,1, \ldots, t$ unabhängig voneinander durchlaufen, kann man zu jedem solchen Paar $v$ und $w$ eine ganze Zahl $u$ bestimmen, so dass

$$
0 \leq u+v \alpha+w \beta<1 \text {. }
$$

Für $u$ gilt also $-t(\alpha+\beta)<u \leq 0$. Von den $(t+1)^{2}$ Zahlen $u+v \alpha+w \beta$ liegen mindestens zwei, $\omega_{1}=u_{1}+v_{1} \alpha+w_{1} \beta$ und $\omega_{2}=u_{2}+v_{2} \alpha+w_{2} \beta$, in einem Intervall der Länge $\left(t^{2}+2 t\right)^{-1}$. Mit $u=u_{1}-u_{2}, v=v_{1}-v_{2}, w=w_{1}-w_{2}$ wird dann, wenn $\omega_{1}>\omega_{2}$,

$$
\begin{aligned}
& 0<u+v \alpha+w \beta<\left(t^{2}+2 t\right)^{-1}, \\
& |u|<t(\alpha+\beta),|v| \leq t,|w| \leq t .
\end{aligned}
$$

Für die so erhaltenen $u, v, w$ ist

$$
\begin{aligned}
\bar{u}+\bar{v} \alpha+\bar{w} \beta & =\frac{1}{2}\left\{(u-v \alpha)^{2}+(u-w \beta)^{2}+(v \alpha-w \beta)^{2}\right\} \\
& <\frac{1}{2} t^{2}\left\{(2 \alpha+\beta)^{2}+(\alpha+2 \beta)^{2}+(\alpha+\beta)^{2}\right\}=t^{2} k
\end{aligned}
$$

mit

$$
k=3 \alpha^{2}+5 \alpha \beta+3 \beta^{2} .
$$

Dann wird

$$
0<F(u, v, w)<t^{2} k\left(t^{2}+2 t\right)^{-1}<k,
$$

und

$$
u+v \alpha+w \beta=\frac{F(u, v, w)}{\bar{u}+\bar{v} \alpha+\bar{w} \beta}>\frac{1}{t^{2} k} .
$$

Wenn jetzt $t^{\prime}$ so gewählt wird, dass $1 /\left(t^{2}+2 t^{\prime}\right)<1 / t^{2} k$, also sicher wenn $t^{\prime} \geq t \sqrt{k}$, bekommt man neue Zahlen $u^{\prime}, v^{\prime}, w^{\prime}$, die (9) genügen. Und mit

$$
t_{v}=([\sqrt{k}]+1)^{p}, \quad v=0,1,2, \ldots,
$$

bekommt man unendlich viele Zahlentripel $u, v, w$, die (9) genügen. 


\section{S. GHRISTOFFERSON, Kubische diophantische Gleichungen mit drei Unbekannten}

Hilfssatz 2. Wenn $\varepsilon=x+y \alpha+z \beta>1$ eine Lösung der Gleichung $F(x, y, z)=1$ ist, sind die Zahlen $x, y, z$ alle positiv.

Beweis. Für die reziproke Lösung $\bar{\varepsilon}$ gilt

$$
0<\bar{x}+\bar{y} \alpha+\bar{z} \beta<1,
$$

und damit können nicht $\bar{x}, \bar{y}, \bar{z}$ alle nichtnegativ oder alle nichtpositiv sein. Wegen $\overline{\bar{\varepsilon}}=\varepsilon$ folgt aus (4) und (7):

$$
\begin{gathered}
x=\bar{x}^{2}-a b \bar{y} \bar{z} \\
y=a \bar{z}^{2}-\bar{x} \bar{y} \\
z=b \bar{y}^{2}-\bar{x} \bar{z} \\
x \bar{x}+a b y \bar{z}+a b z \bar{y}=1 .
\end{gathered}
$$

Aus (7a) folgt sogleich $\bar{x} \neq 0$, und es kann auch nicht $\bar{y}=\bar{z}=0$. Wir können damit folgende vier Fälle behandeln:

1. $\bar{y}=0, \bar{x} \bar{z}<0$ oder $\bar{z}=0, \bar{x} \bar{y}<0$.

Aus (4a), (4b), (4c) folgt $x>0, y>0, z>0$.

2. $\bar{x}>0, \bar{y}>0, \bar{z}<0$ oder $\bar{x}<0, \bar{y}<0, \bar{z}>0$.

Aus (4a), (4c) folgt $x>0, z>0$, und aus (7a) folgt $y>0$.

3. $\bar{x}>0, \bar{y}<0, \bar{z}>0$ oder $\bar{x}<0, \bar{y}>0, \bar{z}<0$.

Aus (4a), (4b) folgt $x>0, y>0$, und aus (7a) folgt $z>0$.

4. $\bar{x}>0, \bar{y}<0, \bar{z}<0$ oder $\bar{x}<0, \bar{y}>0, \bar{z}>0$.

Aus (4b), (4c) folgt $y>0, z>0$, und aus (7a) folgt $x>0$.

\section{§ 3. Existenz und Abschätzung einer Lösung für $C=1$}

Unter den unendlich vielen Zahlentripeln $u, v, w$, die (9) befriedigen, müssen auch unendlich viele $F(u, v, w)$ einen bestimmten Wert $n<3 \alpha^{2}+5 \alpha \beta+3 \beta^{2}$ geben. Und unter diesen letzten Zahlentripeln muss es auch solche geben, für die

$$
u_{i} \equiv u_{j}, v_{i} \equiv v_{j}, w_{i} \equiv w_{j}(\bmod n),
$$

denn die inkongruenten Möglichkeiten sind ja nur $n^{3}$. Dann ist

$$
\begin{gathered}
\frac{u_{i}+v_{i} \alpha+w_{i} \beta}{u_{j}+v_{j} \alpha+w_{j} \beta}=\frac{\left(u_{i}+v_{i} \alpha+w_{i} \beta\right)\left(\bar{u}_{j}+\bar{v}_{j} \alpha+\bar{w}_{j} \beta\right)}{n}=\frac{\xi+\eta \alpha+\zeta \beta}{n} \\
\xi=u_{i} u_{j}^{2}-a b u_{i} v_{j} w_{j}+a b^{2} v_{i} v_{j}^{2}-a b v_{i} u_{j} w_{j}+a^{2} b w_{i} w_{j}^{2}-a b w_{i} u_{j} v_{j} \\
\equiv F\left(u_{i}, v_{i}, w_{i}\right) \equiv 0 \quad(\bmod n) . \\
\eta=a u_{i} w_{j}^{2}-u_{i} u_{j} v_{j}+v_{i} u_{j}^{2}-a b v_{i} v_{j} w_{j}+a b w_{i} v_{j}^{2}-a w_{i} u_{j} w_{j} \equiv 0 \quad(\bmod n) . \\
\zeta=b u_{i} v_{j}^{2}-u_{i} u_{j} w_{j}+a b v_{i} w_{j}^{2}-b v_{i} u_{j} v_{j}+w_{i} u_{j}^{2}-a b w_{i} v_{j} w_{j} \equiv 0 \quad(\bmod n) .
\end{gathered}
$$


Wegen $F(\xi, \eta, \zeta)=n^{3}$ (vgl. (6)), ist dann $x=\xi / n, y=\eta / n, z=\zeta / n$ eine ganzzahlige Lösung der Gleichung

$$
F(x, y, z)=1,
$$

und sie ist von der trivialen Lösung $x=1, y=z=0$ verschieden, weil

$$
u_{i}+v_{i} \alpha+w_{i} \beta \neq u_{j}+v_{j} \alpha+w_{j} \beta .
$$

Der Existenzbeweis ist damit geliefert.

In der Menge von Lösungen $>1$ sind nach Hilfssatz $2 u, v, w$ alle positiv. Also gibt es nur eine endliche Anzahl solche Lösungen unter einer festen Grenze, und daher gibt es auch eine kleinste solche Lösung, $\varepsilon_{1}$, die wir Fundamentallösung der Gleichung (11) nennen.

Wenn $\varepsilon$ eine beliebige Lösung $>1$ von (11) ist, gibt es eine natürliche Zahl $n$, so dass $\varepsilon_{1}^{n} \leq \varepsilon<\varepsilon_{1}^{n+1}$, also

$$
1 \leq \varepsilon \bar{\varepsilon}_{1}^{n}<\varepsilon_{1} .
$$

$\varepsilon \bar{\varepsilon}_{1}^{n}$ ist auch eine Lösung von (11), und wegen der Definition von $\varepsilon_{1}$ muss also $\varepsilon \bar{\varepsilon}_{1}^{n}=1$, und damit

$$
\varepsilon=\varepsilon_{1}^{n}
$$

Sämtliche Lösungen der Gleichung (11) sind also ganzzahlige Potenzen der Fundamentallösung.

Um eine Abschätzung einer Lösung von (11) zu erhalten, benutzen wir den Beweis des Hilfssatzes 1 und den obigen Existenzbeweis, wo wir die Indizes $i$ und $j$ abschätzen wollen.

Mit

$$
N=[k]^{4}
$$

bekommt man für $t_{0}, t_{1}, \ldots, t_{N} N+1$ Werte $0<F(u, v, w) \leqq[k]$. Einer der Werte, $n$, muss mindestens $[k]^{3}+1$ mal angenommen werden. Weil $n \leq[k]$, müssen auch zwei modulo $n$ kongruente Zahlentripel diesen Wert $n$ liefern. Also sind $i, j \leqq N$.

$$
\begin{aligned}
t_{N} & =([\sqrt{k}]+1)^{N} ;\left|u_{i}\right|,\left|u_{j}\right|<t_{N}(\alpha+\beta) ;\left|v_{i}\right|,\left|v_{j}\right|,\left|w_{i}\right|,\left|w_{j}\right| \leqq t_{N} . \\
|x| & =\frac{1}{n}\left|u_{i} \bar{u}_{j}+a b\left(v_{i} \bar{w}_{j}+w_{i} \bar{v}_{j}\right)\right| \\
& <t_{N}^{3}\left\{(\alpha+\beta)\left((\alpha+\beta)^{2}+a b\right)+a b(b+\alpha+\beta+a+\alpha+\beta)\right\}=t_{N}^{3} 2(\alpha+\beta)^{3} .
\end{aligned}
$$

Analoge Abschätzungen von $y$ und $z$ geben, mit $k=3 \alpha^{2}+5 \alpha \beta+3 \beta^{2}$,

$$
\left\{\begin{array}{l}
|x|<2(\alpha+\beta)^{3}([\sqrt{k}]+1)^{[k]^{4}} \\
|y|<14 a^{2}([\sqrt{k}]+1)^{[k]^{4}} \\
|z|<14 a^{2}([\sqrt{k}]+1)^{[k]^{4}}
\end{array}\right.
$$




\section{S. CHRISTOFFERSON, Kubische diophantische Gleichungen mit drei Unbekannten}

Damit ist folgender Satz bewiesen:

Satz 1. a) Die Gleichung $F(x, y, z)=1$ ist immer lösbar.

b) Die Lösungen sind sämtlich gegeben durch die Formel

$$
x_{n}+y_{n} \alpha+z_{n} \beta=\left(x_{1}+y_{1} \alpha+z_{1} \beta\right)^{n}, n=0, \pm 1, \pm 2, \ldots,
$$

wo $x_{1}+y_{1} \alpha+z_{1} \beta$ die kleinste Lösung $>1$ ist.

c) Es gibt mindestens eine Lösung, die den Ungleichungen (12) genügen.

\section{§ 4. Einige Eigenschaften der Lösungen}

Satz 2. Hat $F(u, v, w)=C$ eine Lösung $u+v \alpha+w \beta \geqq \sqrt{C}$, so sind $u, v, w$ alle nichtnegativ.

Beweis. Mit $\omega=u+v \alpha+w \beta>\sqrt{C}$ ist gleichzeitig $\bar{\omega}=\bar{u}+\bar{v} \alpha+\bar{w} \beta<\sqrt{C} . \quad \omega=$ $\sqrt{C}$ gilt nur für $\omega=1, C=1$, und für $C=1$, was im folgenden ausgenommen wird, gilt ja der Satz nach Hilfssatz 2. Der Fall, dass zwei von $u, v, w$ gleich 0 sind, kommt nicht vor, denn dann ist $\omega=\sqrt[3]{C}<\sqrt{C}$, wenn $C>1$. Jetzt wird angenommen, $\omega>\sqrt{C}$ wäre eine Lösung mit mindestens eine der Zahlen $u, v, w$ negativ.

1. $w=0, u v<0 . \bar{\omega}=u^{2}-u v \alpha+b v^{2} \beta=(u+v \alpha)^{2}-3 u v \alpha>\omega^{2}>C$. Widerspruch Entsprechend für $u=0, v w<0$ bzw. $v=0, u w<0$.

2. $\quad u<0, \quad v<0, \quad w>0 . \quad \omega \geqq u^{2}+a b w+a w^{2} \alpha-u v \alpha+b v^{2} \beta+w \beta=(u-v \alpha)^{2}+$ $u v \alpha+a b w+(w \beta)^{2}+w \beta \geqq(w \beta)^{2}>C$. Widerspruch. Entsprechend für $u<0, v>0$, $w<0$ bzw. $u>0, v<0, w<0$.

3. $u<0, v>0, w>0 . \bar{\omega} \geqq u^{2}-a b v w+a w^{2} \alpha+v \alpha+b v^{2} \beta+w \beta=u^{2}+v \alpha+w \beta+$ $(w \beta-v \alpha)^{2}+a b v w>u+v \alpha+w \beta>\sqrt{C}$. Widerspruch. Entsprechend für $v<0$, $u w>0 \mathrm{bzw} . w<0, u v>0$. Damit ist Satz 2 bewiesen.

Wenn $u+v \alpha+w \beta \geqq C$, ist nicht sicher $u v w \neq 0$, wie folgendes Beispiel zeigt: $a b=7 . \quad F(2,1,0)=15.2+\alpha=3,913>3,873=\sqrt{15}$. Dagegen gilt folgender Satz.

Satz 3. Hat $F(u, v, w)=C$ eine Lösung $u+v \alpha+w \beta \geqq \sqrt[3]{4 C}$, so ist $u v w \neq 0$.

Beweis. Wenn $w=0$, ist $u^{3}+(v \alpha)^{3}=C$, und also $v \alpha=\sqrt[3]{C-u^{3}}$. Die Funktion $\omega(u)=u+\sqrt[3]{C-u^{3}}$ nimmt ihren Maximalwert $\sqrt[3]{4 C}$ für $u=\sqrt[3]{C / 2}$ an. Wenn $u=\sqrt{C / 2}$, sind aber $u$ und $v$ keine ganzen Zahlen. Also ist für alle ganzen $u$ und $v$ $u+v \alpha<\sqrt{4 C} . \quad u=0$ bzw. $v=0$ hat dasselbe Resultat zur Folge.

In dem eben erwähnten Beispiel ist $\sqrt[3]{4 C}=\sqrt[3]{60}=3,915$.

Wenn also $\sqrt{C} \geqq \sqrt[3]{4 C}$, folgt aus den beiden letzten Sätzen folgender 
ARKIV FÖR MATEMATIK. Bd 3 nr 30

Satz 4. Hat $F(u, v, w)=C \geqq 16$ eine Lösung $u+v \alpha+w \beta \geqq \sqrt{C}$, so sind $u, v, w$ alle positiv.

Für $C=15$ ist die Behauptung nicht immer richtig, wie das frühere Beispiel zeigt.

Um eine Abschätzung für den Fall, dass $u v w \neq 0$, aber nicht alle $u, v, w>0$, zu erhalten, bemerken wir, dass in diesem Falle genau zwei der Produkte $u v$, $u w, v w$ negativ sind.

$$
2(\bar{u}+\bar{v} \alpha+\bar{w} \beta)=(u-v \alpha)^{2}+(u-w \beta)^{2}+(v \alpha-w \beta)^{2}>(1+\alpha)^{2}+(1+\beta)^{2} .
$$

Also folgt

Satz 5. Hat $F(u, v, w)=C$ eine Lösung, wo $u v w \neq 0$ aber $u, v, w$ nicht alle positiv sind, so gilt

$$
u+v \alpha+w \beta<\frac{2 C}{(1+\alpha)^{2}+(1+\beta)^{2}} .
$$

\section{§ 5. Weitere Eigenschaften der Lösungen}

Wir betrachten die Gleichung (1) für willkürliche positive $u, v, w$. Setzt man

$$
\xi=\frac{u}{w \beta}, \quad \eta=\frac{v \alpha}{w \beta}
$$

wird

$$
\xi^{3}+\eta^{3}+1-3 \xi \eta=\frac{C}{a^{2} b w^{3}} .
$$

Für positive $\xi$ und $\eta$ studieren wir jetzt die Funktion

$$
f(\xi, \eta)=\xi^{3}+\eta^{3}-3 \xi \eta+1 .
$$

Sie hat bei festem $\xi$ ihren kleinsten Wert für $\eta=\sqrt{\varepsilon}$, und dieser Wert ist $\left(\sqrt{\xi^{3}}-1\right)^{2}$. Für eine Lösung von (1) mit positiven $u, v, w$ ist dann

$$
\left(\sqrt{\xi^{3}}-1\right)^{2} \leqq \frac{C}{a^{2} b w^{3}},
$$

oder

Analog wird

$$
\beta\left(1-\sqrt{\frac{C}{a^{2} b w^{3}}}\right)^{\frac{3}{3}} \leqq \frac{u}{w} \leqq \beta\left(1+\sqrt{\frac{C}{a^{2} b w^{3}}}\right)^{\frac{2}{3}} .
$$

und

$$
\frac{\beta}{\alpha}\left(1-\sqrt{\frac{C}{a^{2}} \frac{C}{b w^{3}}}\right)^{\frac{2}{3}} \leqq \frac{v}{w} \leqq \frac{\beta}{\alpha}\left(1+\sqrt{\frac{C}{a^{2} b w^{3}}}\right)^{\frac{3}{3}}
$$

$$
\alpha\left(1-\sqrt{\frac{C}{a b^{2} v^{3}}}\right)^{\frac{8}{3}} \leqq \frac{u}{v} \leqq \alpha\left(1+\sqrt{\frac{C}{a b^{2} v^{3}}}\right)^{\frac{2}{2}} .
$$


S. CHRISTOFFERSON, Kubische diophantische Gleichungen mit drei Unbekannten

Die positiven Lösungen von (1) geben also in gewissem Sinne gute rationale Approximationen der Zahlen $\alpha$ und $\beta$.

Die Gleichheitszeichen können in gewissen Fällen gelten. Ist z. B. $a=n^{3}+1$ und $b=1$, so wird $F\left(n^{2}, n, 1\right)=1$ und

$$
\beta\left(1-\sqrt{\frac{1}{a^{2} b w^{3}}}\right)^{\frac{1}{2}}=\left(n^{3}+1\right)^{\frac{2}{3}}\left(1-\frac{1}{n^{3}+1}\right)^{\frac{3}{3}}=n^{2}=\frac{u}{w}
$$

\section{§ 6. Lösungsklassen und assoziierte Lösungen}

Die Gleichung $F(x, y, z)=1$ ist für jedes $a b$ lösbar, aber es ist nicht immer möglich, ganze Zahlen $u, v, w$ zu finden, die die Gleichung $F(u, v, w)=C, C>1$, befriedigen. Denn eine notwendige Bedingung ist

$$
C \equiv u^{3}(\bmod a) \text { und } C \equiv u^{3}(\bmod b) \text {, }
$$

d.h. $C$ muss kubischer Rest modulo $a$ und $b$ sein.

Angenommen $F(u, v, w)=C$ hat die Lösung

$$
\omega=u+v \alpha+w \beta
$$

dann ist auch (vgl. (5) und (6))

eine Lösung, wo

$$
\omega \varepsilon=u_{1}+v_{1} \alpha+w_{1} \beta
$$

$$
\varepsilon=x+y \propto+z \beta
$$

eine Lösung der Gleichung $F(x, y, z)=1$ ist. Von einer bestimmten Lösung von $F(u, v, w)=C$ ausgehend erhält man also eine unendliche Menge von Lösungen durch Multiplikation mit sämtlichen Lösungen von $F(x, y, z)=1$. Alle so entstandenen Lösungen bilden eine Lösungsklasse, und die Lösungen in einer Klasse nennen wir assoziiert.

Wenn zwei Lösungen $\omega$ und $\omega_{1}$ assoziiert sind, gilt also $\omega_{1}=\omega \varepsilon$ oder $\varepsilon=\omega_{1} / \omega=\omega_{1} \bar{\omega} / C$. Eine notwendige Bedingung ist dann

$$
u_{1} \bar{u}+a b v_{1} \bar{w}+a b w_{1} \bar{v} \equiv u_{1} \bar{v}+v_{1} \bar{u}+a w_{1} \bar{w} \equiv u_{1} \bar{w}+b v_{1} \bar{v}+w_{1} \bar{u} \equiv 0(\bmod C)
$$

Umgekehrt, wenn diese Kongruenzen erfüllt sind, gilt $\omega_{1} \bar{\omega}=C(x+y \alpha+z \beta)$ mit ganzen Zahlen $x, y, z$. Dann ist $C \cdot C^{2}=C^{3} F(x, y, z)$, also $F(x, y, z)=1$, und $\omega_{1}$ und $\omega$ sind assoziiert.

Satz 6. Die notwendige und hinreichende Bedingung dafür, dass die Lösungen $u+v \alpha+w \beta$ und $u_{1}+v_{1} \alpha+w_{1} \beta$ der Gleichung $F(u, v, w)=C$ assoziiert sind, ist, dass die Kongruenzen (13) erfüllt sind. 


\section{§ 7. Abschätzung einer Lösung in einer gegebenen Lösungsklasse}

Mit der Fundamentallösung $\varepsilon$ von $F(x, y, z)=1$ bekannt kann man die Gleichung $F(u, v, w)=C$ vollständig lösen, wenn in jeder Lösungsklasse $K$ eine Lösung bestimmt ist. Wir geben hier eine Abschätzung, mit Hilfe derer man diese Bestimmung machen kann.

Zu jedem $t>0$ gibt es immer eine Lösung in $K$, so dass

Dann ist auch

$$
t \leqq u+v \alpha+w \beta<t \varepsilon \text {. }
$$

$$
\frac{C}{t \varepsilon}<\bar{u}+\bar{v} \alpha+\bar{w} \beta \leqq \frac{C}{t}
$$

Jetzt kann man $t$ so wählen, dass $(t \varepsilon)^{2}=C / t$, d.h.

$$
t=\sqrt[3]{\frac{C}{\varepsilon^{2}}} .
$$

Daraus folgt, dass es eine Lösung $\omega$ in $\boldsymbol{K}$ gibt, so dass

$$
\sqrt[3]{\frac{C}{\varepsilon^{2}}} \leqq \omega<\sqrt[3]{C \varepsilon}
$$

Dieselben Ungleichungen gelten auch für $\left|\omega^{\prime}\right|$ und $\left|\omega^{\prime \prime}\right|$. Diese Abschätzung ist wesentlich besser als die allgemeinere in HECKE [9] und NAGELL [10], wo die obere Grenze $\varepsilon \sqrt[3]{C}$ bzw. $\sqrt{\varepsilon} \sqrt[3]{C}$ ist.

$\mathrm{Da}$ ja $\bar{\omega}=\frac{1}{4}\left\{(2 u-v \alpha-w \beta)^{2}+3(v \alpha-w \beta)^{2}\right\}$, wird

und mit (14)

$$
|2 u-v \alpha-w \beta| \leq 2 \sqrt[3]{C \varepsilon}
$$

$$
\sqrt[3]{\frac{C}{\varepsilon^{2}}}-2 \sqrt[3]{C \varepsilon} \leqq 3 u<3 \sqrt[3]{C \varepsilon}
$$

Wegen der Symmetrie folgt also

Satz 7. In jeder Lösungsklasse der Gleichung $F(u, v, w)=C$ gibt es eine Lösung $u+v \alpha+w \beta$ mit

$$
-\sqrt[3]{C \varepsilon} \frac{2-\bar{\varepsilon}}{3} \leqq u<\sqrt[3]{C \varepsilon}
$$

$v \alpha$ und $w \beta$ liegen zwischen denselben Grenzen.

$\varepsilon$ ist die Fundamentallösung der Gleichung $F(x, y, z)=1$.

Damit ist auch bewiesen, dass die Klassenanzahl endlich ist. 
S. CHRISTOFFERSON, Kubische diophantische Gleichungen mit drei Unbekannten

\section{I T E R A T U R}

I. A. Markoff, Sur les nombres entiers dépendents d'une racine cubique d'un nombre entier. Mém. de l'Acad. Imp. des Sciences de St. Pétersbourg, VII Série, Tome XXXVIII, No. 9 (1882).

2. E. Meisser, Beitrag zur Pell'schen Gleichung höherer Grade. Progr, 283 Ober-Realschıle Kiel (1891).

3. R. DEDEkIND, Über reine kubische Körper. J. f. Math. 121 (1900), 40-123, und Ges. Math. Werke $I I$ (1930), 148-234.

4. T. NAGELL, Über die Einheiten in reinen kubischen Zahlkörpern. Videnskapselskapets skrifter, Christiania (1922).

5. C. Wolfe, On the indeterminate cubic equation $x^{3}+D y^{3}+D^{2} z^{3}-3 D x y z=1$. California Univ. Publ. 16 (1923), 359-369.

6. J. W.S. CAsseLs, The rational solutions of the diophantine equation $Y^{2}=X^{3}-D$. Acta Math. 82 (1950), 243-273.

7. E. S. SelMer, The diophantine equation $\eta^{2}=\xi^{3}-D$. A note on Cassel's method. Math. Scand. 3 (1955), 68-74.

8. G. L. Dirichlet, Werke $I$ (1889), 619-638.

9. E. Hecke, Theorie der algebraischen Zahlen. Leipzig $(1923,1954), 156$.

10. T. NAgELL, Zur algebraischen Zahlentheorie. Math. Zeitschrift 34 (1931), 191. 